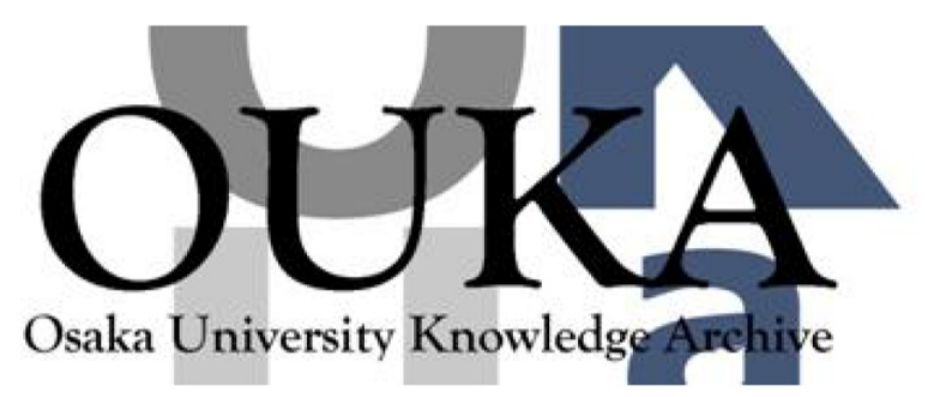

\begin{tabular}{|c|c|}
\hline Title & $\begin{array}{l}\text { Cathodoluminescence from Organic Bi layer } \\
\text { Induced by Field Electron Emission of Carbon } \\
\text { Nanotubes }\end{array}$ \\
\hline Author (s) & $\begin{array}{l}\text { Nanjundaswamy, Rashmi; Williams, Christopher; } \\
\text { Lee, Sergey B. et al. }\end{array}$ \\
\hline Citation & 電気材料技術雑誌. 14(2) p. 75-p. 78 \\
\hline Issue Date & $2005-07-15$ \\
\hline oaire:version & VoR \\
\hline URL & https://hdl. handle. net/11094/76809 \\
\hline rights & \\
\hline Note & \\
\hline
\end{tabular}

Osaka University Knowledge Archive : OUKA

https://ir. Library. osaka-u. ac. jp/

Osaka University 
電気材料技術雑誌 第 14 巻第 2 号

J.Soc.Elect.Mat.Eng. Vol.14, No.2, 2005

\title{
Cathodoluminescence from Organic Bilayer Induced by Field Electron Emission of Carbon Nanotubes
}

\author{
Rashmi Nanjundaswamy, Christopher Williams, Sergey B. Lee, Anvar A. Zakhidov \\ Department of Physics and Nanotech Institute, University of Texas at Dallas, Richardson, TX
}

Received 4 January 2005; accepted 8 January 2005

\begin{abstract}
We report the observation and studies of cathodoluminescence (CL) of organic bilayer structures, such as tris-(8-hydroxyquinoline) aluminium (Alq3) and 2-(4biphenyl)-5-(4-t-butylphenyl)-1,3,4-oxadiazole (PBD) deposited on ITO-coated glass, with and without hole transport layer, $N, N^{\prime}$-diphenyl- $N, N^{\prime}$-bis(1-naphthyl)-(1,1'-phenyl)-4,4'-diamine ( $\alpha$-NPD). To excite the CL of such bilayer organic heterostructure, low energy electrons field emitted by single walled carbon nanotube cathodes were utilized. The dependence of $C L$ spectrum and intensity on voltage (V), current (I), type of transport layer and the cathode-anode geometry has been studied. We propose carbon nanotubes as efficient cathodes for stable CL emission from organic multi-layer heterostructures. The role of both electron and hole transport layers on stability and spectrum of $\mathrm{CL}$ is also discussed.
\end{abstract}

Keywords: cathodoluminescence, field emission, electroluminescence, exciplex, organic light-emitting diods

\section{Introduction}

Electroluminescence (EL) of organic materials has been investigated very actively since the development of very efficient organic light emitting diodes (OLED) and polymeric organic light emitting diodes (POLED) due to their potential application for lighting and flat panel displays [1]. Much of recent efforts have been devoted to improvement and optimization of performance of organic devices. To achieve this goal, the balanced injection of electrons and holes with subsequent radiative recombination of singlet or triplet excitons or exciplexes is needed. The simple approach is to use low work function electrodes for electron injection and high work function electrodes for hole injection. A more advanced approach is the formation of a $p-i-n$ structure with $n$ - and $p$-doped transport layers [2]. This approach works perfectly well only for p-type doping, i.e. for hole injection. However, $n$ type doping of organic layers (under stable medium work function electrode, e.g Al) is done by using a very thin layer of $\mathrm{Li}$ [3] or by direct $\mathrm{Li}$ doping [2] which is not stable in ambient atmosphere.

When the energy of injected carriers becomes much higher than the gap Eg, several interesting phenomena may occur: The hot charge carrier injection at very high electric fields and the effect of impact ionization was observed in POLED [4,5] and OLED [6]. Such an approach is especially interesting for future realization of electrically pumped organic lasers. If the energy of injected electrons is sufficient enough, they can cause impact ionization and charge carrier multiplication. The threshold for impact ionization in poly(p-phenylenevinylene) (PPV) was estimated to be about $3.7 \mathrm{eV}$ [5]. Chayet and co-workers have observed new UV emission in PPV due to direct radiative transition above threshold voltage of $\sim 100 \mathrm{~V}$ [4]. This new feature increases exponentially with the applied field above the threshold field. The same phenomena have been observed in Alq3 thin films [6].

Since their discovery in 1991 [7], carbon nanotubes (CNT) have emerged as a promising candidates for thin film carbon nanotube cold cathodes for field emission flat panel display and light source applications due to their peculiar structural and electronic properties $[8,9]$. Because of the low threshold field and high current density of field emission from carbon nanotubes, they are an ideal electron source to study the $C L$ in organic materials.

Cathodoluminescence of inorganic phosphors is used in CNT-based field emission display [10]. However, to best of our knowledge, cathodoluminescence of organic materials has not been studied as actively as electroluminescence. 
電気材料技街雑誌 第 14 巻第 2 号

J.Soc.Elect.Mat.Eng. Vol.14, No.2, 2005
Mostly, electron microscopists use cathodoluminescence of organic matter for imaging polymer and organic molecular thin films [11]. There were experimental attempts in former Soviet Union to obtain electron beam-pumped lasing from complex organic molecules in the condensed phase [12]. An interesting study of local EL and CL spectra from thin films of the conjugated polymer (soluble PPV derivative), generated by an electron beam from the STM tip has been reported [13]. The CL spectra, very similar to PL and EL, were observed at electric field of $200 \mathrm{~V} / \mu \mathrm{m}$.

In this paper, we report our studies of CL emission from organic bilayers induced by field emitted electrons from carbon nanotube cold cathodes. The motivation is to clarify how interfaces between charge transport and emissive layers effect the $\mathrm{CL}$ spectrum. Particularly interesting problem we address here is how injection of holes from ITO substrate of our multilayers into hole transport layer changes the $\mathrm{CL}$.

\section{Experiment}

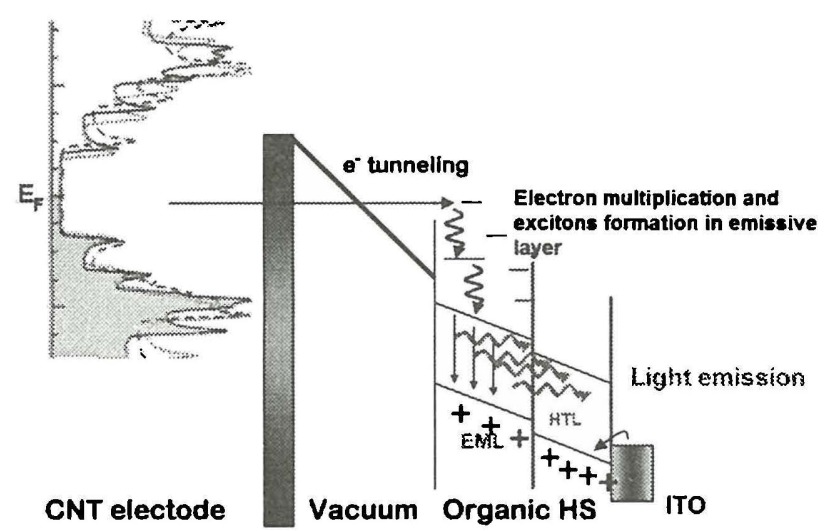

Fig. 1. A schematic of the field-emission electron injection from CNT cathode into two layer OLED and light emission process. On the left side of the figure the electronic states of CNT cathode are shown. The shaded area shows the occupied states below the Fermi energy which are contributing to the field emission current. The contribution from singularities below the Fermi energy can enhance the field emission current significantly.

The schematic energy diagram of OLED with CNT field emission cathode is shown in Fig. 1. In OLED the exciton formation is the result of electron and hole injection from the opposite cathode and anode, respectively. In CL the electron injection occurs from the CNT field emitter.

Field emission current was obtained from purified single walled carbon nanotube paper. HiPCO-type single wall carbon nanotubes were obtained from Carbon Nanotechnologies, Inc. The free standing nanotube sheets were prepared by vacuum filtration of $\sim 20 \mathrm{ml}$ of a $\sim 0.6$ $\mathrm{mg} / \mathrm{ml}$ nanotube suspension through a $47 \mathrm{~mm}$ diameter poly-(tetra-fluoroethylene) filter (Millipore LS, $5 \mu \mathrm{m}$ pores). The film was washed several times with $200 \mathrm{ml}$ of de-ionized water, followed by $100 \mathrm{ml}$ of methanol, to remove residual $\mathrm{NaOH}$ and surfactant, respectively. These sheets were allowed to dry under continuous vacuum pumping for 1 hour before being peeled off the PTFE filter.
The paper was then purified. An SEM image of the carbon nanotube paper is shown in Fig. 2.

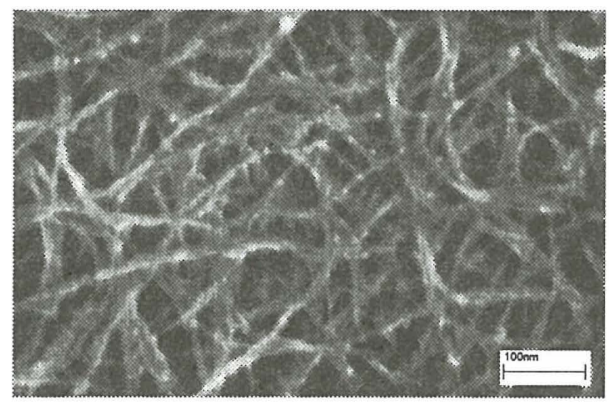

Fig. 2. SEM image of SWNT paper. Scale bar $100 \mathrm{~nm}$.

Organic thin films were deposited onto Corning 1737 glass substrates coated with indium tin oxide $(5-15 \Omega / \square)$ (Delta Technologies, Inc.). ITO substrates were cleaned using proper solvents and treated in oxygen plasma prior to film deposition. Thin films were deposited using thermal evaporation at a base pressure $<2 \times 10^{-6}$ Torr. Tris-(8hydroxyquinoline) aluminum (Alq3), a green emitting material and 2-Phenyl-5-(4-biphenylyl)-1,3,4-oxadiazole (PBD), which is a blue emitting material, were used as the light emitting layers. PBD is also an electron transport material, i.e. a hole-blocking layer, and $N, N^{\prime}$-diphenyl$N, N^{\prime}$-bis(1-naphthyl)-(1,1'-phenyl)-4,4'-diamine ( $\alpha$-NPD) is a hole transport layer. The following layers and bilayers were prepared: ITO/Alq ${ }_{3}(500 \AA)$; ITO $\alpha$ $\mathrm{NPD}(700 \AA) / \mathrm{Alq}_{3}(500 \AA) ; \mathrm{ITO} / \alpha-\mathrm{NPD}(700 \AA) / \mathrm{PBD}(500 \AA)$. Similar OLED structures with $\mathrm{Al}(1000 \AA)$ cathodes instead of CNT were also prepared for comparison.

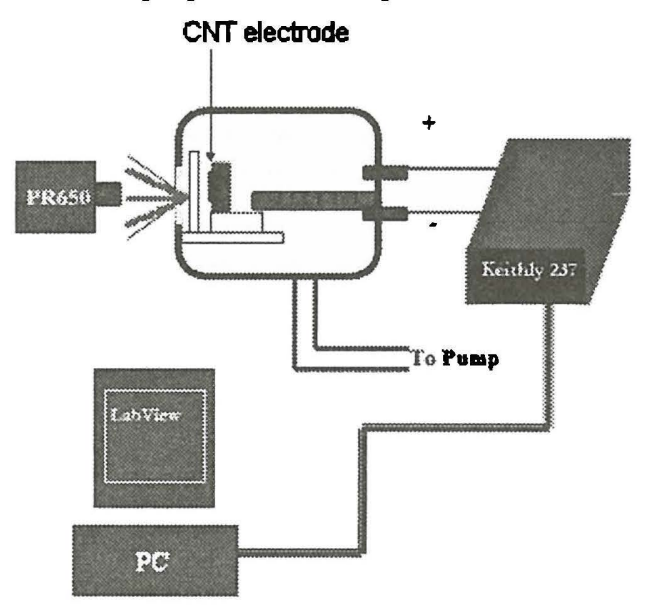

Fig. 3. Experimental setup.

Organic layers were deposited at a rate of $1 \AA / s$, and organic bilayers were deposited in succession without breaking vacuum. The $\mathrm{CL}$ and field emission measurements were performed in a high vacuum chamber equipped with a turbo-molecular pump. A diode setup was used for performing the experiments (see Fig. 3). Both anode and cathode mounting stands were made of aluminium and were hand ground to achieve perfectly parallel surfaces. The anode-mounting stand was fixed on a 
slide, and a micrometer gauge was used for adjusting the position of the cathode and thus the anode-cathode spacing. The single wall carbon nanotubes paper was mounted on the cathode stand and the ITO glass coated with organic layers was mounted on the anode. A glass window in the vacuum chamber facilitated the spectral measurement of the emitted light with the PR650 spectroradiometer (PhotoResearch, Inc.). Current-voltage characteristics were measured using Keithley 237 high voltage source-measure unit (sweep mode).

\section{Results and discussion}

Fig. 4 shows both the typical plot of the field emission current as a function of applied field (inset) and the FowlerNordheim plot for the CNT paper. The threshold average electric field at which electron emission can be detected is as low as $1.2 \mathrm{~V} / \mu \mathrm{m}$.

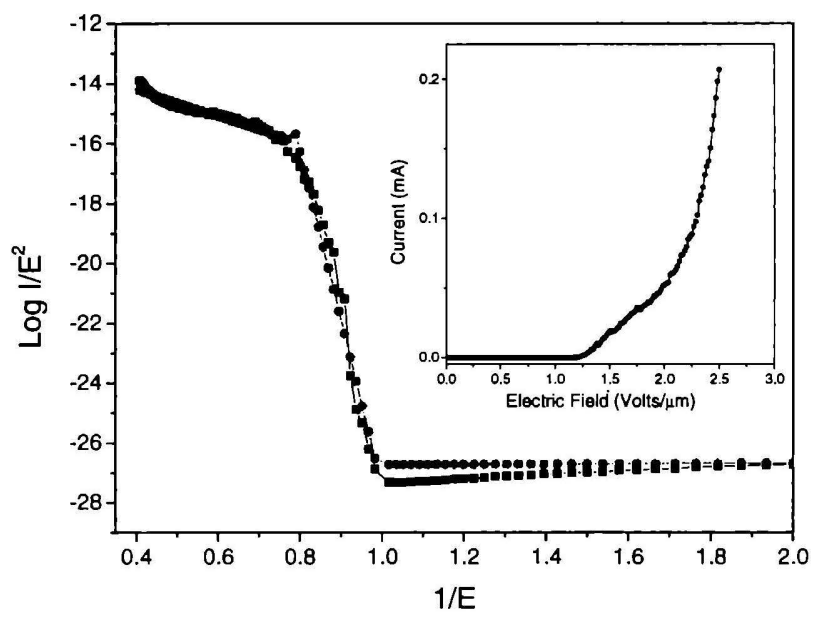

Fig. 4 Fowler-Nordheim plot.

Inset shows current vs $\mathrm{E}$ dependence.

Fig. 5 shows normalized intensity of $\mathrm{CL}$ and EL for both the Alq $3 / \alpha-N P D / T T O$ and PBD/ $\alpha$-NPD/ITO structures. The CL spectra were measured at constant electric field of $2.5 \mathrm{~V} / \mu \mathrm{m}(500 \mathrm{~V}, 200 \mu \mathrm{m})$ and $2.1 \mathrm{~V} / \mu \mathrm{m}(420 \mathrm{~V}, 200 \mu \mathrm{m})$ for Alq3 and PBD, respectively. EL spectra were measured from the bilayer comparison OLEDs at an applied bias of 7 $\mathrm{V}$.

Fig.5a shows the comparison spectra of Alq3 with $\alpha$ NPD as the hole transport layer. The maximum of main spectral peak emitted is the same for both EL and CL and is centered at $2.35 \mathrm{eV}(528 \mathrm{~nm})$. A photograph of green light emission from Alq3 with a hole-transport layer is also shown in the inset of Fig.5a. The emission pattern maps the highly emissive spots of CNT electrode.

In Fig 5b a comparison of CL and EL spectra for PBD with $\alpha-$ NPD as the hole transport layer is shown. The peaks of EL and CL are shifted from each other by $40 \mathrm{~nm}$. Samples that do not have $\alpha-N P D$ or another hole transport layer emit very low intensity CL light.
Energy diagrams for both the Alq3/ $\alpha-\mathrm{NPD} / \mathrm{ITO}$ and $\mathrm{PBD} / \alpha-N \mathrm{ND} / \mathrm{ITO}$ are shown in Fig. 6 . The electron affinity and the ionization potential of $\alpha$-NPD has been reported to be $2.3 \mathrm{eV}$ and $5.4 \pm 0.1 \mathrm{eV}$, correspondingly, and the ionization energy of Alq3 was measured to be $5.8 \pm 0.1 \mathrm{eV}$ [14]. The electron affinity of Alq3 was estimated to be 3.1 $\mathrm{eV}$ using the optical energy gap of Alq3 $(2.7 \mathrm{eV})$.

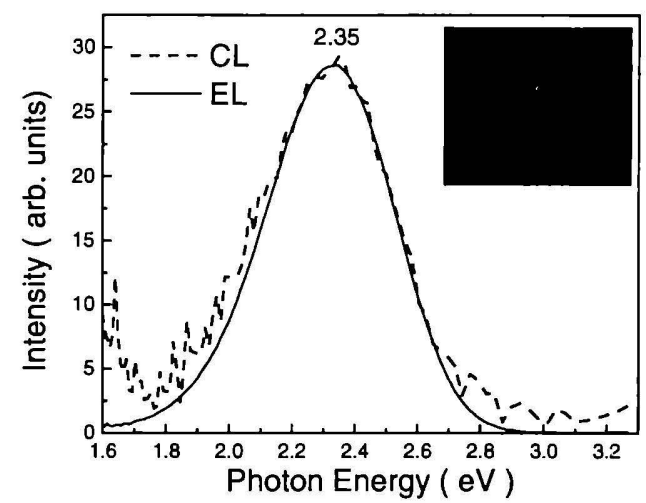

a)

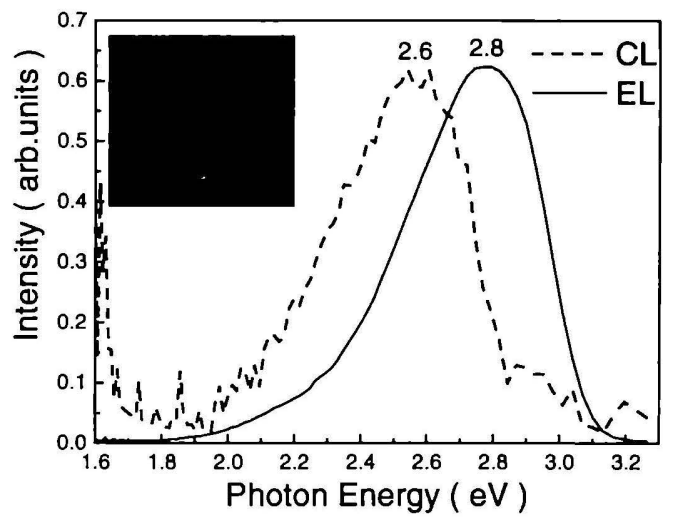

b)

Fig. 5 a) CL spectra from an Alq3/ $\alpha-N P D / I T O$ device (measured at 2.3 $\mathrm{V} / \mu \mathrm{m})$ and $\mathrm{EL}$ of an Al/Alq3/ $\alpha-\mathrm{NPD} / \mathrm{ITO}$ device. Inset: Optical digital image of CL from an Alq3/ $\alpha$-NPD/ITO device. b) Spectra of CL from a PBD/ $\alpha$-NPD/ITO device (measured at $2.1 \mathrm{~V} / \mu \mathrm{m}$ ) and EL of an $\mathrm{Al} / \mathrm{PBD} / \alpha-\mathrm{NPD} / \mathrm{ITO}$ device. Inset: Optical digital image of $\mathrm{CL}$ from a PBD/ $\alpha-\mathrm{NPD} / \mathrm{ITO}$ device.

In the case of Alq3/ $\alpha$-NPD/TTO, the hot injected electrons decay to the ground state of Alq3 (Fig.1) and are trapped within Alq3 layer because of electron-blocking effect of $\alpha$-NPD. The $0.8 \mathrm{eV}$ offset is a significant barrier for electrons. In contrast, there is no significant barrier $(0.4$ $\mathrm{eV}$ ) for holes injected through $\alpha$-NPD from ITO. Although the voltage drops mostly in the vacuum gap between the CNT field emitter and organic layer, we believe that there is still some voltage drop at the organic layer/ITO interface, and this drop is sufficient for hole injection from ITO. Hence, the Alq3 emission was observed in both cases of Fig. 5,a).

The situation is completely different in the case of 
$\mathrm{PBD} / \alpha-\mathrm{NPD} / \mathrm{TO}$. PBD, with a very high ionization potential (6.1-6.2 eV) forms a hole blocking barrier (0.7-0.8 $\mathrm{eV})$. The holes are trapped at the interface of PBD with the $\alpha$-NPD layer. The electrons are efficiently injected from the CNT emitter. The EL from $\alpha-\mathrm{NPD}$ is dominant $(2.8 \mathrm{eV}$ or $440 \mathrm{~nm}$ ), although there is a long tail in visible range. We believe that there is a contribution from exciplex formation at the interface between PBD and $\alpha$-NPD.

The most interesting experimental observation is the red shift (from $2.8 \mathrm{eV}$ to $2.6 \mathrm{eV}$ ) of $\mathrm{CL}$ spectrum from $\mathrm{PBD} / \alpha-\mathrm{NPD} / \mathrm{TTO}$ structure. We didn't observe a blue shift due to direct interband transitions from Alq3 or PBD or $\alpha$ NPD as was reported [6], probably, because of moderate electric field $\left(-2 \times 10^{4} \mathrm{~V} / \mathrm{cm}\right)$ applied during measurement. The photo-luminescence maximum for PBD is at $3.26 \mathrm{eV}$ $(380 \mathrm{~nm})$ [15] which are much higher than the observed emission peak. We don't know about experimental observations of electroluminescence from PBD. Therefore, we attribute the red shift to exciplex emission at the PBD/ $\alpha$-NPD interface (Fig.6b). Highly efficient red-shifted exciplex emission has indeed been observed at various interfaces with PBD $[15,16]$ and $\alpha$-NPD [17]. This exciplex emission may be very promising for solid state organic lasers electrically pumped by high intensity electron emission from CNT electrodes because it will not suffer from light re-absorption problems.

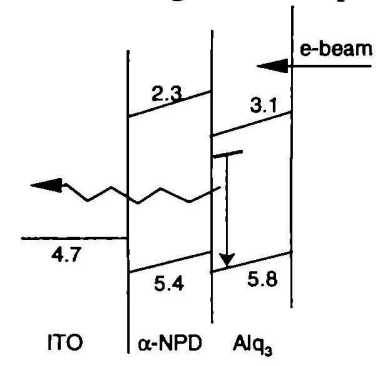

a)

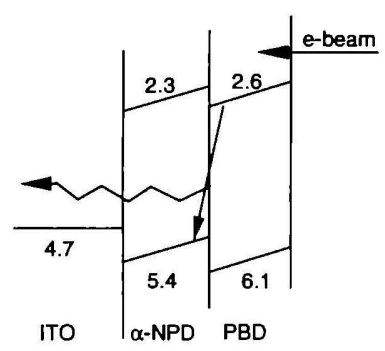

b)
Fig. 6. Energy diagrams of bilayer structures Alq3/ $\alpha-\mathrm{NPD} / \mathrm{TTO}$ and PBD/ $\alpha-\mathrm{NPD} / \mathrm{TTO}$

In conclusion, we should discuss the stability of organic materials interacting with an energetic electron beam. To avoid radiation damage to the organic layer we use low energy $[\leq 0.5 \mathrm{keV}]$ electron injection with a short mean free path. One of the dominant processes causing radiation damage in organic materials is carbon $\mathrm{K}$-shell ionization which is quite high $(285 \mathrm{eV})$. Very low energy e-beams do not cause serious damage to aromatic molecules [18], so we don't expect serious radiation damage in our case. Drummy and co-workers [11] have estimated the mean free path for total electron scattering to be $15 \mathrm{~nm}$ for organic samples at $5 \mathrm{kV}$. Such a small mean free path enables them to obtain high contrast images from very thin organic films. We expect a much lower value for mean free path in our experiment. Short penetration depth of low energy electrons is advantageous for thin film OLED devices.

The more serious problem for field emission cathodes is non-uniformity of field emission from CNT paper and
Joule heating.. We have found that as DC current density increases the organic layer heats up and sublimes onto the cathode. Short high voltage pulses with low duty cycle should be used to minimize heating and sublimation of organic materials [19].

In summary, we have reported efficient electron injection from a CNT cathode to an organic bilayer LED structure. Strong cathodoluminescence, enhanced by hole injection into $\alpha$-NPD followed by radiative recombination of singlet excitons in the Alq 3 layer of the Alq $3 / \alpha$ NPD/TO structure has been observed. Cathodoluminescence due to exciplex emission at the $\mathrm{PBD} / \alpha-\mathrm{NPD}$ interface has been observed in $\mathrm{PBD} / \alpha-$ NPD/ITO structures. It is not clear at this stage why the CL spectrum of the PBD/ $\alpha-\mathrm{NPD} / \mathrm{ITO}$ structure shows exciplex emission while EL is dominated by exciton emission from $\alpha-\mathrm{NPD}$. We should remind that the measurements were made at a low applied electric field. The brightest redshifted CL emission was observed at the edges of the CNT paper, i.e. at the highest electric field, where a blue-shifted emission is expected. Further experimental study is needed to clarify the problem. The electric field strength between the electrodes can reach very high values up to $10^{7} \mathrm{~V} / \mathrm{cm}$. At such high fields the excited states can be formed by direct electron impact ionization. In addition, the electron excitation is less selective than optical excitation [12]. Therefore, arbitrary states of organic molecules can be excited by an electron beam of sufficient energy. We believe that comparatively more excitons can be formed as a result of charge multiplication in the organic layers in this CL process.

Acknowledgements We would like to thank SPRING Consortium and the Genesis Campus, USA for the partial funding, and Roman Kikta for continued support.

\section{References}

[1] Organic Light-Emitting Devices, J.Shinar, Ed., Springer, 2003

[2] J. Huang et al., Appl. Phys. Lett. 80 (2002) 139.

[3] G. Parthasarathy, et.al J. Appl. Phys. 89 (2001) 4986.

[4] H. Chayet, R. Pogreb, D. Davydov, Phys. Rev. B 56 (1997) R12702.

[5] E.M. Conwell, Phys. Rev. B 57 (1998) R 12670.

[6] X.L. Xu, J. Appl. Phys. 89 (2001) 1082.

[7] S.lijima, Nature 354 (1991) 56.

[8] Y.Saito, J. Nanosci. Nanotech., 3 (2003) 39.

[9] A.N. Obraztsov, , in Perspectives of Fullerene Nanotechnology, E.Osawa, eds. (2002) 67.

[10] J.M. Kim et al., Diamond and Related Materials, 9 (2000) 1184

[11] L.F.Drummy, J.Yang, D.C. Martin, Ultramicroscopy 99 (2004) 247.

[12] A.V. Kukhto, J. Appl. Spectroscopy 65 (1998) 722.

[13] D.G. Lidzey et al, Nature 386 (1997) 135; D.G. Lidzey et al,, Appl. Phys. Lett. 71 (1997) 2008.

[14] I.G. Hill and A. Kahn, J.Appl.Phys. 86 (1999) 4515.

[15] X.Jiang et al., J.Appl.Phys. 91 (2002) 6717.

[16] Y. Kawabe and J. Abe, Appl. Phys. Lett. 81 (2002) 493.

[17] L.C. Palilis, et.al.,, Appl. Phys. Lett. 82 (2003) 2209.

[18] A. Howie, F.J. Rocca, U. Valdre, Phil. Mag. B52 (1986) 71.

[19] J.R. Fryer, Ultramicrocopy 23 (1987) 321 